\title{
Lie Symmetries and Criticality of Semilinear Differential Systems
}

\author{
Yuri BOZHKOV ${ }^{\dagger}$ and Enzo MITIDIERI ${ }^{\ddagger}$ \\ † Departamento de Matemática Aplicada, Instituto de Matemática, \\ Estatistica e Computação Científica, Universidade Estadual de Campinas - UNICAMP, \\ C.P. 6065, 13083-970 - Campinas - SP, Brasil \\ E-mail: bozhkov@ime.unicamp.br \\ URL: http://www.ime.unicamp.br/ bozhkov/ \\ ¥Dipartimento di Matematica e Informatica, Università degli Studi di Trieste, \\ Via Valerio 12/1, 34127 Trieste, Italia \\ E-mail: mitidier@units.it \\ URL: http://www.dmi.units.it/ mitidier/
}

Received February 01, 2007, in final form March 20, 2007; Published online March 25, 2007

Original article is available at http://www.emis.de/journals/SIGMA/2007/053/

\begin{abstract}
We discuss the notion of criticality of semilinear differential equations and systems, its relations to scaling transformations and the Noether approach to Pokhozhaev's identities. For this purpose we propose a definition for criticality based on the S. Lie symmetry theory. We show that this definition is compatible with the well-known notion of critical exponent by considering various examples. We also review some related recent papers.
\end{abstract}

Key words: Pokhozhaev identities; Noether identity; critical exponents

2000 Mathematics Subject Classification: 35J50; 35J20; 35J60; 35L70

\section{Introduction}

It is well known that the so-called critical exponents are found as critical powers for embedding theorems of Sobolev type. They can be also viewed as numbers which divide existence and nonexistence of solutions for various semilinear differential equations and systems involving power nonlinearities. Such equations appear when the Frechét derivatives of Sobolev and $L_{p}$ norms are considered.

The aim of this paper is to discuss a notion of criticality of differential equations, its relations to scaling transformations and the Noether approach to Pokhozhaev's identities [13]. Our interpretation is based on the S. Lie symmetry theory of differential equations $[5,29,30,24]$ and the criticality is considered in terms of group invariance. In this sense we propose a definition of criticality. Then we show that it is compatible with the notion of critical exponent by considering various examples. Although these examples are semilinear differential equations and systems, we believe that this point of view can trace new directions and provide deeper understanding of more general differential equations and systems.

To begin with, we recall some results already discussed in [6]. Let us first consider the following class of ordinary differential equations for $v=v(r), r>0$ :

$$
-\left(r^{\alpha}\left|v^{\prime}(r)\right|^{\beta} v^{\prime}(r)\right)=\mu r^{\gamma}|v(r)|^{p-1} v(r),
$$

where $\alpha, \beta, \gamma, \mu$ and $p$ are real numbers and $v^{\prime}=\frac{d v}{d r}$. This class was introduced in [4] and studied by Clemént, de Figueiredo and Mitidieri in [15]. It contains various differential equations, e.g. 
the equations which are radial forms of PDE involving the Laplace, $p$-Laplace or $k$-Hessian operators, the Lane-Emden equation, Emden-Fowler equation, etc. Such equations come from mechanics, astrophysics, general relativity, theories of gravitation, atomic physics and quantum mechanics. We shall assume that the parameters satisfy the inequalities

$$
\mu>0, \quad \beta>-1, \quad \alpha-\beta-1>0,
$$

and

$$
\beta+1<p \leq q^{*}-1
$$

where

$$
q^{*}=\frac{(\gamma+1)(\beta+2)}{\alpha-\beta-1}
$$

is the critical exponent for (1.1). See [15].

For this class it has been observed in [10] that a Lie point symmetry of (1.1) is a variational symmetry if and only if $p+1=q^{*}$, the critical exponent. This fact suggests that the critical exponent for (1.1) may as well be defined as the only exponent for which any Lie point symmetry of (1.1) is a variational symmetry. This is a way to define the critical exponent without involving functional analysis. That is, using directly the ordinary differential equation which occurs in the most cases when reducing the proof of the embedding theorems to radially symmetric functions. We shall come back to this point later. We just observe that for the radial form

$$
\varphi^{\prime \prime}+\frac{n-1}{r} \varphi^{\prime}+|\varphi|^{p-1} \varphi=0
$$

of the partial differential equation

$$
\Delta u+|u|^{p-1} u=0
$$

in $\mathbb{R}^{n}, n \geq 3$, we have $\alpha=\gamma=n-1, \beta=0$ and the critical exponent is exactly

$$
p=\frac{n+2}{n-2},
$$

the well-known Sobolev exponent. The latter property explains why the Lane-Emden equation

$$
v^{\prime \prime}+\frac{2}{r} v^{\prime}+v^{5}=0,
$$

for $v=v(r)$, describing a star as a ball of condensed gases, can be explicitly solved (since the critical exponent in spatial dimension 3 is exactly 5 and hence any Lie point symmetry is variational reducing the order of integration procedure by two). We also recall that the equation (1.2) admits positive solutions if and only if $p \geq(n+2) /(n-2)$. See [20,14].

The symmetry approach was applied in [11] to the radial Lane-Emden system in $\mathbb{R}^{n}, n \geq 3$,

$$
\begin{aligned}
& u^{\prime \prime}+\frac{n-1}{2} u^{\prime}+|v|^{q-1} v=0, \\
& v^{\prime \prime}+\frac{n-1}{2} v^{\prime}+|u|^{p-1} u=0 .
\end{aligned}
$$

The obtained result states that a Lie point symmetry is a variational symmetry of (1.3) if and only if the positive numbers $p$ and $q$ are such that the point $(p, q)$ belongs to the critical hyperbola

$$
\frac{1}{p+1}+\frac{1}{q+1}=\frac{n-2}{n} \text {. }
$$


By the results of Mitidieri [25, 26, 27] and Serrin-Zou [36, 37, 38] it follows that (1.4) divides existence and nonexistence of positive solutions of (1.3). Hence the name critical hyperbola. Related existence and nonexistence results for higher order equations and systems are contained in $[32,34,39]$ and [28]. For further details concerning Noether symmetries for (1.1) and (1.3) see the review paper [6] and the references therein. We point out that similar ideas and results pertaining to semilinear wave equations have appeared in the recent papers [1] and [2].

One can observe that in all these cases the symmetries are dilations of the independent and the dependent variables. The scaling transformations of this type play an important role since the invariance with respect to such transformations is equivalent to an application of a dimensional analysis argument [5, 29].

In this paper we shall show that similar properties are valid for partial differential equations and systems which have a variational structure. This will allow to define the notion of criticality for such differential equations and systems. For this purpose we consider the Euler-Lagrange equations

$$
E(L)=0
$$

for the functional

$$
J[u]=\int_{\Omega} L\left(x, u^{\alpha}, u_{(k)}^{\alpha}\right) d x
$$

where $x \in \Omega \subseteq \mathbb{R}^{n}, n \geq 1, u^{\alpha}(x), \alpha=1,2, \ldots, m$, are $C^{k}(\Omega)$ functions, $k \geq 1$, the Function of Lagrange $L=L\left(x, u^{\alpha}, u_{(k)}^{\alpha}\right)$ depends on $x, u^{\alpha}$ and the partial derivatives of $u^{\alpha}$ up to order $k$, and $E=\left(E_{1}, \ldots, E_{m}\right)$ is the Euler operator.

Now we present the basic definition regarding the criticality of the systems (1.5) in the semilinear case.

Definition 1. Suppose that (1.5) is a semilinear system. We say that it is critical if there exists a dilation

$$
X=a_{i} x_{i} \frac{\partial}{\partial x_{i}}+b^{\alpha} u^{\alpha} \frac{\partial}{\partial u^{\alpha}}
$$

such that

$$
X^{(k)} L+L \sum_{i=0}^{n} a_{i}=0
$$

where $a_{i}, i=1, \ldots, n, b^{\alpha}, \alpha=1,2, \ldots, m$, are real constants and $X^{(k)}$ is the $k$-th order prolongation of $X$.

Above and throughout this paper we assume summation over a repeated index: the Latin indices vary from 1 to $n$, while the Greek ones - from 1 to $m$.

The first immediate observation is that the relation (1.8) means that $X$ is a variational symmetry, that is a symmetry of the action functional $(1.6)[5,29,30]$. This conclusion follows from the infinitesimal criterion of invariance $[29,30]$. In this way it is clear that the left-hand side of (1.8) is the left-hand side of the Noether identity [24, 23] for the particular case of dilations $X$. The Noether identity was discussed in [13] and used to obtain Pokhozhaev type identities. The choice of critical values in the Noether identity allows to obtain the 'right' form of the Pokhozhaev's identity [13] and the corresponding nonexistence results in appropriate functional spaces for problems which obey certain type of homogeneity.

We note that in the present paper we shall deal only with dilations which, as we shall show, cover the known cases of critical semilinear differential equations and systems. Indeed, all 
considered examples admit dilations as symmetries. In regard to more general differential equations and systems another possible definition of criticality based on the property established and discussed in $[10,11,6,7]$ relating the variational/divergence symmetries of critical differential equations and the Sobolev theorem would be the following

Definition 2. We say that the system (1.5) is critical if any its generalized (Lie-Bäcklund) symmetry is variational or divergence symmetry.

However, in order to apply the Definition 2 one needs a complete group classification of the considered differential equations or systems which for the present is not available for some of the examples. The group analysis of these cases itself is a subject of another work and applications of Definition 2 will be treated elsewhere.

By a straightforward calculation of the extended infinitesimals of the dilation $X$ one can see that in more detail the equation (1.8) reads

$$
\begin{aligned}
& a_{i} x_{i} \frac{\partial L}{\partial x_{i}}+b^{\alpha} u^{\alpha} \frac{\partial L}{\partial u^{\alpha}}+\left(b^{\alpha}-a_{i}\right) u_{i}^{\alpha} \frac{\partial L}{\partial u_{i}^{\alpha}}+\cdots \\
& \quad+\left(b^{\alpha}-\sum_{s=1}^{k} a_{i_{s}}\right) u_{i_{1} i_{2} \ldots i_{k}}^{\alpha} \frac{\partial L}{\partial u_{i_{1} i_{2} \ldots i_{k}}^{\alpha}}+L \sum_{i=0}^{n} a_{i}=0 .
\end{aligned}
$$

Although the general solution of the first order linear partial differential equation (1.9) can be easily found, we shall proceed in a different way. Namely, we shall consider various concrete differential equations and systems, and for each of them we shall find the criticality condition in terms of its specific parameters. Typical examples are the following theorems, which are among the main new results obtained in the present paper.

Theorem 1. Let $F=F\left(u^{1}, \ldots, u^{m}\right) \in C^{1}\left(\mathbb{R}^{m}\right)$. Then the system

$$
\begin{aligned}
& -\Delta u^{1}=F_{u^{1}}, \\
& -\Delta u^{2}=F_{u^{2}}, \\
& \cdots \cdots \cdots \cdots \\
& -\Delta u^{m}=F_{u^{m}}
\end{aligned}
$$

is critical if and only if

$$
\sum_{i=1}^{m} u^{i} F_{u^{i}}=\sigma F, \quad \text { where } \quad \sigma=\frac{2 n}{n-2} .
$$

Observe that the equality (1.11) is the Euler identity for $F$. Hence we have

Corollary 1. The potential system (1.10) is critical if and only if $F$ is a homogeneous function of degree $\sigma=\frac{2 n}{n-2}$.

A further result can be stated as

Theorem 2. Let $H=H\left(u^{1}, \ldots, u^{m}, v^{1}, \ldots, v^{m}\right) \in C^{1}\left(\mathbb{R}^{m}\right)$. Then the system of $2 m$ equations

$$
\begin{aligned}
& -\Delta u^{1}=H_{v^{1}}, \\
& -\Delta v^{1}=H_{u^{1}}, \\
& \cdots \cdots \cdots \cdots \\
& -\Delta u^{m}=H_{v^{m}} \\
& -\Delta v^{m}=H_{u^{m}}
\end{aligned}
$$


is critical if and only if

$$
a^{\alpha} u^{\alpha} H_{u^{\alpha}}+\left(1-a^{\alpha}\right) v^{\alpha} H_{v^{\alpha}}=\theta H
$$

where $\theta=\frac{n}{n-2}$ and $a^{\alpha}, \alpha=1, \ldots, m$, are real positive constants.

Corollary 2. The system (1.12) is critical if and only if $H=H\left(u^{1}, \ldots, u^{m}, v^{1}, \ldots, v^{m}\right)$ is a sum of homogeneous functions and identity (1.13) holds.

In this paper we adopt the following terminology. Let $L$ be a linear elliptic differential operator in divergence form and $L^{*}$ - its formally adjoint operator. An Euler-Lagrange system of type

$$
L u=F_{u}(u, v), \quad L^{*} v=F_{v}(u, v)
$$

will be called elliptic potential system, while a system of the form

$$
L u=H_{v}(u, v), \quad L^{*} v=H_{u}(u, v)
$$

will be called elliptic Hamiltonian system. (Such terminology has been used in analysis, e.g. [16] and [17].) Thus the system (1.10) is an elliptic potential system and the system (1.12) is an elliptic Hamiltonian system.

Nonexistence results for Hamiltonian systems (1.12) were obtained by Mitidieri in [25, 26, 27] using Rellich type identities established in the same articles. See also [39].

This work is a natural continuation of the preceding one [6] presented at the 6th International Conference "Symmetry in Nonlinear Mathematical Physics", June 20-26, 2005, Kyiv, Ukraine. Here in Sections 4, 6, 8 we shall illuminate some more or less known results. The results obtained in Sections 7, 9-13 are new. We shall also review some recent papers [13, 8, 9]. In particular, in Section 3, we shall comment on the role of the critical exponents in establishing of Pokhozhaev's identities which will complement the discussion in [13]. The exposition in some parts follows closely the text of the original articles. It corresponds to the talk one of us (Y.B.) is going to give during the 7th International Conference "Symmetry in Nonlinear Mathematical Physics", June 24-30, 2007, Kyiv, Ukraine.

This paper is organized as follows. In the next section we introduce notations and preliminaries. Then in Section 3 we comment on the Noether approach to Pokhozhaev identities [13]. In the subsequent sections we apply the basic definition to the following partial differential equations and systems: nonlinear Poisson equations, $p$-Laplace equations, equations involving polyharmonic, Baouendi-Grushin and Kohn-Laplace operators, elliptic systems of potential, Hamiltonian and mixed type, hyperbolic Hamiltonian systems and unbounded Hamiltonian systems. For each case we find the corresponding criticality conditions. If the nonlinearities are of power type we show that the proposed definition is compatible with the notions of critical exponent and critical hyperbola. In this regard we consider some model equations and systems. Theorems 1 and 2 are proved in Sections 9 and 10 respectively.

\section{Preliminaries}

In this section we outline very briefly some basic notions and formulae regarding variational properties of differential equations and systems as well as Lie groups generators and their extensions. For further details, systematic and profound expositions the interested reader is directed to $[5,24,29,30]$.

We shall suppose that all considered functions, vector fields, tensors, functionals, etc. are sufficiently smooth in order for the derivatives we write to exist. The independent variable 
$x \in \Omega \subseteq \mathbb{R}^{n}$ - a bounded or unbounded domain. In this work we are mainly interested in group invariance properties of the considered differential equations and systems. For this reason we shall not treat the boundary terms and the regularity of solutions.

The partial derivatives of a smooth function $v=v(x)$ are denoted by subscripts:

$$
v_{i}:=\frac{\partial v}{\partial x_{i}}, \quad v_{i j}:=\frac{\partial^{2} v}{\partial x_{i} \partial x_{j}},
$$

etc. We shall also assume summation over a repeated index. The Latin indices vary from 1 to $n$, while the Greek ones - from 1 to $m$. The latter will denote collections of functions, e.g. $v^{\alpha}(x)$.

We introduce the total derivative operator

$$
D_{i}=\frac{\partial}{\partial x_{i}}+u_{i}^{\alpha} \frac{\partial}{\partial u^{\alpha}}+u_{i j}^{\alpha} \frac{\partial}{\partial u_{j}^{\alpha}}+\cdots+u_{i i_{1} i_{2} \ldots i_{l}}^{\alpha} \frac{\partial}{\partial u_{i_{1} i_{2} \ldots i_{l}}^{\alpha}}+\cdots,
$$

where $u^{\alpha}(x)$ are given functions. (See [5, 29].) If $v$ is a function of $x, u^{\alpha}$ and the derivatives of $u^{\alpha}$ up to order $k$, then

$$
D_{i} v=\frac{\partial v}{\partial x_{i}}+u_{i}^{\alpha} \frac{\partial v}{\partial u^{\alpha}}+u_{i j}^{\alpha} \frac{\partial v}{\partial u_{j}^{\alpha}}+\cdots+u_{i i_{1} i_{2} \ldots i_{l}}^{\alpha} \frac{\partial v}{\partial u_{i_{1} i_{2} \ldots i_{k}}^{\alpha}} .
$$

The Euler-Lagrange equations, corresponding to the functional

$$
J[u]=\int_{\Omega} L\left(x, u^{\alpha}, u_{(k)}^{\alpha}\right) d x
$$

are given by

$$
E_{\alpha}(L)=\frac{\partial L}{\partial u^{\alpha}}-D_{i} \frac{\partial L}{\partial u_{i}^{\alpha}}+D_{i} D_{j} \frac{\partial L}{\partial u_{i j}^{\alpha}}+\cdots+(-1)^{k} D_{i_{1}} D_{i_{2}} \cdots D_{i_{k}} \frac{\partial L}{\partial u_{i_{1} i_{2} \ldots i_{k}}^{\alpha}}=0,
$$

where the operator

$$
E_{\alpha}=\frac{\partial}{\partial u^{\alpha}}-D_{i} \frac{\partial}{\partial u_{i}^{\alpha}}+D_{i} D_{j} \frac{\partial}{\partial u_{i j}^{\alpha}}+\cdots+(-1)^{k} D_{i_{1}} D_{i_{2}} \cdots D_{i_{k}} \frac{\partial}{\partial u_{i_{1} i_{2} \ldots i_{k}}^{\alpha}}+\cdots
$$

is the $\alpha$-th component of the Euler operator $E=\left(E_{1}, \ldots, E_{m}\right)$ which corresponds to the (unknown) function $u^{\alpha}$. See [5, 29].

Further, consider the differential operator

$$
X=\xi^{i} \frac{\partial}{\partial x_{i}}+\eta^{\alpha} \frac{\partial}{\partial u^{\alpha}} .
$$

The functions

$$
\xi^{i}=\xi^{i}(x, u)=\xi^{i}\left(x_{1}, \ldots, x_{n}, u^{1}, \ldots, u^{m}\right)
$$

and

$$
\eta^{\alpha}=\eta^{\alpha}(x, u)=\eta^{\alpha}\left(x_{1}, \ldots, x_{n}, u^{1}, \ldots, u^{m}\right)
$$

are called infinitesimals of the one-parametric group of point transformations generated by $X$, that is the transformation

$$
x_{j}^{*}=x_{j}^{*}(x, u, \varepsilon), \quad u^{* \alpha}=u^{* \alpha}(x, u, \varepsilon),
$$


where $\varepsilon$ is a parameter and

$$
\xi^{i}=\left.\frac{\partial x_{i}^{*}}{\partial \varepsilon}\right|_{\varepsilon=0}, \quad \eta^{\alpha}=\left.\frac{\partial u^{* \alpha}}{\partial \varepsilon}\right|_{\varepsilon=0} .
$$

Given a transformation (2.2) one can calculate $\xi^{i}$ and $\eta^{\alpha}$ by (2.3). And vice-versa, given $x_{j}, u^{\alpha}$, $\xi^{i}$ and $\eta^{\alpha}$, the one-parametric group of point transformations (2.2) is determined by the unique solution of the problem

$$
\begin{array}{ll}
\frac{d x_{j}^{*}}{d \varepsilon}=\xi^{j}\left(x_{i}^{*}, u^{* \alpha}\right), & \frac{d u^{* \alpha}}{d \varepsilon}=\eta^{\alpha}\left(x_{i}^{*}, u^{* \alpha}\right), \\
\left.x_{j}^{*}\right|_{\varepsilon=0}=x_{j}, & \left.u^{* \alpha}\right|_{\varepsilon=0}=u^{\alpha} .
\end{array}
$$

Henceforth we shall identify the Lie point transformation (2.2) and its infinitesimal generator $X$.

We associate to $X$ its $k$-th order prolongation $X^{(k)}$ given by

$$
X^{(k)}=\xi^{i} \frac{\partial}{\partial x_{i}}+\eta \frac{\partial}{\partial u}+\eta_{i}^{(1) \alpha} \frac{\partial}{\partial u_{i}^{\alpha}}+\cdots+\eta_{i_{1} i_{2} \ldots i_{k}}^{(k) \alpha} \frac{\partial}{\partial u_{i_{1} i_{2} \ldots i_{k}}^{\alpha}},
$$

where

$$
\begin{aligned}
& \eta_{i}^{(1) \alpha}=D_{i} \eta^{\alpha}-\left(D_{i} \xi^{j}\right) u_{j}^{\alpha}, \quad i=1,2, \ldots, n ; \\
& \eta_{i_{1} i_{2} \ldots i_{l}}^{(l) \alpha}=D_{i_{l}} \eta_{i_{1} i_{2} \ldots i_{l-1}}^{(l-1) \alpha}-\left(D_{i_{l}} \xi^{j}\right) u_{i_{1} i_{2} \ldots i_{l-1} j}^{\alpha},
\end{aligned}
$$

with $i_{l}=1,2, \ldots, n$ for $l=2,3, \ldots, k, k=2,3, \ldots$ See $[5,29]$ for further details. The functions $\eta_{i_{1} i_{2} \ldots i_{l}}^{(l) \alpha}$ are called extended infinitesimals.

Definition 3. A vector field $X$ is a divergence symmetry of $J[u]$ if there exists a vector function $B=\left(B_{1}, B_{2}, \ldots, B_{n}\right)$ of $x, u$ and its derivatives up to some finite order, such that

$$
X^{(m)} L+L D_{i} \xi^{i}=D_{i} B_{i}
$$

or equivalently,

$$
\begin{aligned}
\frac{\partial L}{\partial x_{i}} \xi_{i} & +\frac{\partial L}{\partial u} \eta+\frac{\partial L}{\partial u_{i}}\left(D_{i} \eta-u_{j} D_{i} \xi_{j}\right)+\cdots \\
& +\frac{\partial L}{\partial u_{i_{1} i_{2} \ldots i_{m}}}\left[D_{i_{1}} D_{i_{2}} \cdots D_{i_{m}}\left(\eta-u_{j} \xi_{j}\right)+\xi_{j} u_{j i_{1} i_{2} \ldots i_{m}}\right]+L D_{i} \xi^{i}=D_{i} B_{i} .
\end{aligned}
$$

If $B=0$, then $X$ is called variational symmetry.

Hence, clearly the relation (1.8) means that the dilation (1.7) is a variational symmetry.

\section{On the Noether approach to Pokhozhaev identities}

The celebrated Pokhozhaev's identity [31, 32] is an important tool in the theory of differential equations. Among a big variety of applications, it is particularly useful in establishing of nonexistence results. Commonly its specific form for each concrete problem is obtained by using ad hoc procedures.

In [13] we have recently proposed a general unified method to generate Pokhozhaev identities. This approach is based on the Noether identity and the Lie symmetry theory. It has been applied in [13] to various nonlinear differential equations and systems choosing transformation parameters assuming critical values. The essential points of this method can be summarized as follows. 
Let $u^{\alpha}(x), \alpha=1,2, \ldots, m$, be a set of $C^{2 k}(\Omega)$ functions, where $k \geq 1$ and $x \in \Omega \subseteq \mathbb{R}^{n}, n \geq 1$. We denote by $A_{k}$ the space of all locally analytic functions of $x, u^{\alpha}$ and the partial derivatives of $u^{\alpha}$ up to order $k$. The elements $f\left(x, u^{\alpha}, u_{(k)}^{\alpha}\right)$ of $A_{k}$ are called differential functions [5, 24, 29].

Consider a differential operator of the form

$$
X=\xi^{i} \frac{\partial}{\partial x_{i}}+\eta^{\alpha} \frac{\partial}{\partial u^{\alpha}}
$$

where $\xi^{i}, \eta^{\alpha} \in A_{k}$. Let

$$
L=L\left(x, u^{\alpha}, u_{(k)}^{\alpha}\right) \in A_{k}
$$

be an arbitrary differential function. Then the following identity holds

$$
X^{(k)} L+L D_{i} \xi^{i}=E_{\alpha}(L)\left(\eta^{\alpha}-u_{j}^{\alpha} \xi^{j}\right)+D_{i}\left[L \xi^{i}+W_{i}\left[u, \eta-u_{j} \xi^{j}\right]\right],
$$

where $u=\left(u^{1}, \ldots, u^{m}\right), \eta=\left(\eta^{1}, \ldots, \eta^{m}\right), u_{i}=\left(\frac{\partial u^{1}}{\partial x_{i}}, \ldots, \frac{\partial u^{m}}{\partial x_{i}}\right), X^{(k)}$ is the $k$-th order prolongation of $X, E=\left(E_{1}, \ldots, E_{m}\right)$ is the Euler operator and the operator $W_{i}$ is defined in [5].

The identity (3.1) is called the Noether identity [24, 23]. It is the corner stone of the approach suggested in [13]. The main point of [13] is the observation that the Pokhozhaev's identity for solutions of differential equations can be obtained from the Noether identity for functions after integration and application of the Gauss-Ostrogradskii theorem, with account of the boundary conditions.

It is clear that the crucial step in establishing of the Pokhozhaev identities is the choice of the operator $X$ which appears in (3.1). For the semilinear differential equations and systems considered in [13] $X$ was a dilation whose parameters assume critical values. In the present work we show how to find such critical parameters. Actually, the use of critical values of the equation parameters in obtaining the Pokhozhaev's identities is the main motivation to write this paper.

\section{Nonlinear Poisson equations}

Let $x \in \mathbb{R}^{n}, n \geq 3$. It is well known that the equation

$$
\Delta u+f(u)=0
$$

has a variational structure. Its function of Lagrange is given by

$$
L=\frac{1}{2} u_{j}^{2}-F(u), \quad F(u)=\int_{0}^{u} f(z) d z .
$$

We shall look for a constant $a$ such that

$$
X^{(1)} L+n L=0,
$$

where

$$
X=x_{i} \frac{\partial}{\partial x_{i}}+a u \frac{\partial}{\partial u}
$$

and the first order prolongation of $X$ is given by

$$
X^{(1)}=x_{i} \frac{\partial}{\partial x_{i}}+a u \frac{\partial}{\partial u}+(a-1) u_{i} \frac{\partial}{\partial u_{i}} .
$$


By a straightforward calculation

$$
X^{(1)} L+n L=\left(a+\frac{n-2}{2}\right) u_{j}^{2}-a u f(u)-n F(u)
$$

Let $a=(2-n) / 2$. Then by (4.2) the equation (4.1) is critical if and only if

$$
\frac{n-2}{2} u f(u)-n F(u)=0 \text {. }
$$

Hence we have proved

Theorem 3. The equation (4.1) is critical if and only if

$$
f(u)=c|u|^{2^{*}-1} u,
$$

where $2^{*}=2 n /(n-2)$ and $c$ is an arbitrary constant.

For the equation (4.1) in a bounded domain $\Omega \subset R^{n}, n \geq 3$, with homogeneous Dirichlet condition $u=0$ on $\partial \Omega$, S.I. Pokhozhaev [31] obtained in 1965 the following identity

$$
\int_{\Omega}\left[\frac{n-2}{2} u f(u)-n F(u)\right] d x=-\frac{1}{2} \int_{\partial \Omega}|\nabla u|^{2}(x, \nu) d s,
$$

where $\nu$ is the outward unit normal to $\partial \Omega$. This identity immediately follows from (4.2) with $a=(2-n) / 2$ and the Noether identity.

\section{$5 \quad p$-Laplace equations}

An argument similar to that presented in the preceding section applies to quasilinear equations involving the $p$-Laplace operator $\Delta_{p}, p<n$, given by $\Delta_{p} u:=\operatorname{div}\left(|\nabla u|^{p-2} \nabla u\right)$. The result states:

Theorem 4. The equation

$$
\Delta_{p} u+|u|^{p^{*}-1} u=0
$$

in $\mathbb{R}^{n}$, where $n>p$ and $p^{*}=n p /(n-p)$ is the unique critical quasilinear $p$-Laplace equation. (The uniqueness is up to multiplying factors of $u$.)

Remark. The above equation should be interpreted in a suitable weak form. For identities related to the equation

$$
\Delta_{p} u+f(u)=0
$$

where $f: \mathbb{R} \rightarrow \mathbb{R}$ is a given function, we refer the interested reader to [22] where a Pokhozhaev identity for $C^{1, \alpha}$-solutions is obtained. See also [34].

\section{Polyharmonic equations}

In this section we consider the polyharmonic equation

$$
(-\Delta)^{k} u+f(u)=0
$$


in $\mathbb{R}^{n}, n>2 k$. It was shown in [7] that the dilation

$$
Z=x_{i} \frac{\partial}{\partial x_{i}}+\frac{2 k}{1-p} u \frac{\partial}{\partial u}
$$

is a variational symmetry of

$$
(-\Delta)^{k} u+|u|^{p-1} u=0
$$

if and only if

$$
p=\frac{n+2 k}{n-2 k},
$$

the critical Sobolev exponent. In this case all symmetries of (6.2) are divergence symmetries [7].

Let $k$ be an even number. Then, as is well known, (6.1) is the Euler-Lagrange equation of the functional

$$
\int L=\int\left[\frac{1}{2}\left|\Delta^{k / 2} u\right|^{2}-F(u)\right] d x, \quad F(u)=\int_{0}^{u} f(z) d z .
$$

Thus the equation (1.9) with $m=1, u^{1}=u, a^{1}=a$ assumes the following form:

$$
a u \frac{\partial L}{\partial u}+(a-k) u_{i_{1} i_{2} \ldots i_{k}} \frac{\partial L}{\partial u_{i_{1} i_{2} \ldots i_{k}}}+n L=0
$$

since $L$ does not depend on the derivatives of $u$ of order less than $k$. Substituting

$$
\frac{\partial L}{\partial u_{i_{1} i_{2} \ldots i_{k}}}=\left(\Delta^{k / 2} u\right) \delta_{i_{1} i_{2}} \cdots \delta_{i_{k-1} i_{k}}
$$

into (6.4) we obtain

$$
\left(a-k+\frac{n}{2}\right)\left(\Delta^{k / 2} u\right)^{2}-a u f(u)-n F(u)=0 .
$$

We choose $a=(2 k-n) / 2$. Then, by (6.5), the equation (6.1) is critical if and only if

$$
\frac{n-2 k}{2} u f(u)-n F(u)=0
$$

Hence (6.1) is critical if and only if

$$
f(u)=c u^{\frac{n+2 k}{n-2 k}}
$$

where $c$ is an arbitrary constant. Thus the following theorem holds:

Theorem 5. The equation (6.2) with p given by (6.3) is the only critical semilinear polyharmonic equation (since the constant $c$ can be incorporated into $u$ by the change of the dependent variable $u=\mu v$ with $\left.\mu=c^{(2 k-n) /(4 k)}\right)$.

The case $k$-odd can be treated in a similar way.

For important results related to (6.1) see [34] and [35]. 


\section{Baouendi-Grushin equations}

Let $x \in \mathbb{R}^{n}, y \in \mathbb{R}^{m}, n \geq 1, m \geq 1$ and $u=u(x, y) \in C^{2}\left(\mathbb{R}^{n} \times \mathbb{R}^{m}\right)$ be a scalar function. Let $\alpha>0$ be a real number. Then the generalized Baouendi-Grushin operator [3, 21] is defined by

$$
\Delta_{L} u=\Delta_{x} u+|x|^{2 \alpha} \Delta_{y} u
$$

where $\Delta_{x} u=u_{x_{i} x_{i}}$ and $\Delta_{y} u=u_{y_{\mu} y_{\mu}}$ are the standard Laplacians in $\mathbb{R}^{n}$ and $\mathbb{R}^{m}$ respectively, and $|x|=\left(x_{i}^{2}\right)^{1 / 2}$.

For recent results and applications of the Baouendi-Grushin operator see [18] and the references therein. We just recall here that the critical exponent associated to $\Delta_{L}$ is $\frac{Q+2}{Q-2}$, where $Q=n+(\alpha+1) m>2$ is the so-called homogeneous dimension.

We consider the following semilinear equation:

$$
\Delta_{L} u+|u|^{p-1} u=0 .
$$

Formally it is the Euler-Lagrange equation of the functional $J[u]=\int F$ where the function of Lagrange is given by:

$$
F=\frac{1}{2}\left|\nabla_{x} u\right|^{2}+\frac{1}{2}|x|^{2 \alpha}\left|\nabla_{y} u\right|^{2}-\frac{1}{p+1}|u|^{p+1}=\frac{1}{2} u_{x_{i}}^{2}+\frac{1}{2}|x|^{2 \alpha} u_{y_{\mu}}^{2}-\frac{1}{p+1}|u|^{p+1} .
$$

It is easy to see by a straightforward calculation that the dilation

$$
x_{j}^{*}=\lambda x_{j}, \quad y_{\mu}^{*}=\lambda^{\alpha+1} y_{\mu}, \quad u^{*}=\lambda^{2 /(1-p)} u,
$$

is admitted by the Baouendi-Grushin equation (7.2), that is, it is a Lie point symmetry of (7.2). Then using the infinitesimal criterion of invariance [29] or performing in the action integral $J[u]$ the above change of variables, we obtain that the dilation (7.3) is a variational symmetry of (7.2) if and only if

$$
p=\frac{Q+2}{Q-2} .
$$

Clearly, if $\alpha=0$ this is the critical Sobolev exponent.

Now we shall study the criticality of the equation

$$
\Delta_{L} u+f(u)=0 .
$$

We aim to clarify for which functions $f$ this equation would be critical. For this purpose we consider the dilation

$$
X=x_{i} \frac{\partial}{\partial x_{i}}+(\alpha+1) y_{\mu} \frac{\partial}{\partial y_{\mu}}+a u \frac{\partial}{\partial u},
$$

where $a$ is a constant to be determined. Then the equation (1.9) assumes the following form:

$$
\left(a-1+\frac{Q}{2}\right)\left|\nabla_{x} u\right|^{2}+\left(a-1+\frac{Q}{2}\right)|x|^{2 \alpha}\left|\nabla_{y} u\right|^{2}-a u f(u)-Q F(u)=0 .
$$

We choose $a=(2-Q) / 2$. Then (7.4) is critical if and only if

$$
\frac{Q-2}{2} u f(u)-Q F(u)=0 .
$$

That is, $f(u)=c u^{(Q+2) /(Q-2)}$. The following theorem summarizes the above considerations.

Theorem 6. Up to some multiplying factors, the equation

$$
\Delta_{L} u+u^{(Q+2) /(Q-2)}=0
$$

is the only critical semilinear partial differential equation involving the Baouendi-Grushin operator. 


\section{Kohn-Laplace equations}

In this section we shall review and comment on some results obtained in [8,9].

As it is well known the Heisenberg group $H^{n}$ topologically is the real vector space $\mathbb{R}^{2 n+1}$. endowed with the product

$$
(x, y, t)\left(x^{1}, y^{1}, t^{1}\right)=\left(x+x^{1}, y+y^{1}, t+t^{1}+2 \sum_{i=1}^{n}\left(y_{i} x_{i}^{1}-x_{i} y_{i}^{1}\right)\right),
$$

where $(x, y, t),\left(x^{1}, y^{1}, t^{1}\right) \in \mathbb{R}^{n} \times \mathbb{R}^{n} \times \mathbb{R}=H^{n}$. In the last few decades a significant number of works treats partial differential equations on the Heisenberg group $H^{n}$. In this regard various authors have obtained existence and nonexistence results for equations involving Kohn-Laplace operators. The following equation

$$
\Delta_{H^{n}} u+f(u)=0,
$$

or equivalently

$$
u_{x_{i} x_{i}}+u_{y_{i} y_{i}}+4\left(x_{i}^{2}+y_{i}^{2}\right) u_{t t}+4 y_{i} u_{x_{i} t}-4 x_{i} u_{y_{i} t}+f(u)=0
$$

will be called the Kohn-Laplace equation. Here the Kohn-Laplace operator $\Delta_{H^{n}}$ is the natural subelliptic Laplacian on $H^{n}$ defined by

$$
\Delta_{H^{n}}=\sum_{i=1}^{n}\left(X_{i}^{2}+Y_{i}^{2}\right)
$$

where

$$
X_{i}=\frac{\partial}{\partial x_{i}}+2 y \frac{\partial}{\partial t}, \quad Y_{i}=\frac{\partial}{\partial y_{i}}-2 x \frac{\partial}{\partial t} .
$$

Recall that in [19] Garofalo and Lanconelli established existence, regularity and nonexistence results for the Kohn-Laplace equation in an open bounded or unbounded subset of $H^{n}$ with homogeneous Dirichlet boundary condition. The existence of weak solutions is proved in [19] provided the nonlinear term satisfies some growth conditions of the form $f(u)=o\left(|u|^{(Q+2) /(Q-2)}\right)$ as $|u| \rightarrow \infty$, where $Q=2 n+2$ is the homogeneous dimension of $H^{n}$ ([19]). The exponent $(Q+2) /(Q-2)$ is the critical exponent for the Stein's Sobolev space [19]. The nonexistence results follow from remarkable Pokhozhaev identities established in [19] for the solutions of Kohn-Laplace equations on the Heisenberg group. General nonexistence results for solutions of semilinear differential inequalities on the Heisenberg group were obtained by Pokhozhaev and Veron in [33]. In [8] a complete group classification of Kohn-Laplace equations on $H^{1}$ is carried out.

We observe that the Kohn-Laplace equation is formally the Euler-Lagrange equation of the functional

$$
J[u]=\int L,
$$

with

$$
\begin{aligned}
L & =\frac{1}{2}\left(X_{i} u\right)^{2}+\frac{1}{2}\left(Y_{i} u\right)^{2}-\int_{0}^{u} f(s) d s \\
& =\frac{1}{2} u_{x_{i}}^{2}+\frac{1}{2} u_{y_{i}}^{2}+2\left(x_{i}^{2}+y_{i}^{2}\right) u_{t}^{2}+2 y_{i} u_{x_{i}} u_{t}-2 x_{i} u_{y_{i}} u_{t}-\int_{0}^{u} f(s) d s .
\end{aligned}
$$


Then using the definition of Lie point symmetry of a differential equation, one can show that the scaling transformation

$$
x_{j}^{*}=\lambda x_{j}, \quad y_{j}^{*}=\lambda y_{j}, \quad t^{*}=\lambda^{2} t, \quad u^{*}=\lambda^{\frac{2}{1-p}} u
$$

is admitted by the equation

$$
\Delta_{H^{n}} u+|u|^{p-1} u=0 .
$$

Further, following [9] and performing this change of variables in the functional $J$, it is easy to see that the dilation

$$
Z=x_{i} \frac{\partial}{\partial x_{i}}+y_{i} \frac{\partial}{\partial y_{i}}+2 t \frac{\partial}{\partial t}+\frac{2}{1-p} u \frac{\partial}{\partial u}
$$

is a variational symmetry if and only if

$$
p=\frac{n+2}{n}=\frac{Q+2}{Q-2} .
$$

Thus the equation (8.2) admits the variational symmetry group generated by $Z$ if and only if $p$ assumes the critical value. Hence one concludes as in Sections 3 and 5 that the following theorem holds.

Theorem 7. The equation (8.2) with $p=(n+2) / n$ is the only critical Kohn-Laplace equation.

\section{$9 \quad$ Elliptic potential systems}

In this section we prove Theorem 1.

The function of Lagrange for the potential system (1.10) is given by

$$
L=\frac{1}{2} u_{j}^{\alpha} u_{j}^{\alpha}-F\left(u^{1}, \ldots, u^{m}\right) .
$$

Let $a$ be a constant and consider a dilation of the form

$$
X=x_{i} \frac{\partial}{\partial x_{i}}+a u^{\alpha} \frac{\partial}{\partial u^{\alpha}} .
$$

Our aim is to find out a constant $a$ such that $X$ is a variational symmetry of (1.10), and, hence (1.10) would be critical (see Introduction). Substituting (9.1) into the equation (1.9) we obtain that (1.10) is critical if and only if

$$
\left(a-1+\frac{n}{2}\right) u_{j}^{\alpha} u_{j}^{\alpha}-a u^{\alpha} F_{u^{\alpha}}-n F=0 .
$$

Choosing $a=(2-n) / 2$ we conclude from (9.2) that (1.10) is critical if and only if (1.11) holds.

\section{Elliptic Hamiltonian systems}

In this section we prove Theorem 2 and some corollaries.

The Function of Lagrange for the Hamiltonian system (1.12) is given by

$$
L=\frac{1}{2} u_{j}^{\alpha} v_{j}^{\alpha}-H\left(u^{1}, \ldots, u^{m}, v^{1}, \ldots, v^{m}\right) .
$$


In order to satisfy the basic definition (see Introduction) we shall look for a dilation of type

$$
X=x_{i} \frac{\partial}{\partial x_{i}}+A^{\alpha} u^{\alpha} \frac{\partial}{\partial u^{\alpha}}+B^{\alpha} v^{\alpha} \frac{\partial}{\partial v^{\alpha}}
$$

where $A^{\alpha}, B^{\alpha}, \alpha=1, \ldots, m$, are constants to be determined later. By (1.9) and (10.1) we obtain:

$$
X^{(1)} L+n L=\left(A^{\alpha}+B^{\alpha}-2+n\right) u_{j}^{\alpha} v_{j}^{\alpha}-A^{\alpha} u^{\alpha} H_{u^{\alpha}}-B^{\alpha} v^{\alpha} H_{v^{\alpha}}-n H .
$$

Let $A^{\alpha}+B^{\alpha}=2-n$ for $\alpha=1, \ldots, m$. Then by (10.3) and the Definition 1 , the Hamiltonian system (1.12) is critical if and only if

$$
-A^{\alpha} u^{\alpha} H_{u^{\alpha}}-B^{\alpha} v^{\alpha} H_{v^{\alpha}}=n H
$$

which implies (1.13) if we denote $a^{\alpha}=-A^{\alpha}(n-2)$. This completes the proof of Theorem 2 .

Further we consider the particular case $m=1, u^{1}=u, v^{1}=v, a^{\alpha}=a$. The condition (1.13) reads

$$
a u H_{u}+(1-a) v H_{v}=\theta H .
$$

The general solution of this linear first order partial differential equation is

$$
H=u^{\theta / a} \phi\left(u^{1-a} v^{-a}\right)
$$

where $\phi$ is an arbitrary function and $\theta=n /(n-2)$. If

$$
H=\frac{1}{q+1}|u|^{q-1} u+\frac{1}{p+1}|v|^{p-1} v
$$

by (10.4) we have that the corresponding Lane-Emden system

$$
-\Delta u=|v|^{p-1} v, \quad-\Delta v=|u|^{q-1} u,
$$

is critical if and only if

$$
a u^{q+1}+(1-a) v^{p+1}=\frac{n}{n-2}\left(\frac{1}{q+1} u^{q+1}+\frac{1}{p+1} v^{p+1}\right) .
$$

Hence the following theorem holds:

Theorem 8. The system (10.5) is critical if and only if

$$
\frac{1}{p+1}+\frac{1}{q+1}=\frac{n-2}{n}
$$

that is, if and only if $(p, q)$ belongs to the critical hyperbola (1.4).

\section{Mixed systems}

Analogously to the previous two sections we prove 
Theorem 9. The mixed Hamiltonian-potential system consisting of $2 m+r$ equations

$$
\begin{aligned}
& -\Delta u^{1}=H_{v^{1}}, \\
& -\Delta v^{1}=H_{u^{1}}, \\
& \cdots \cdots \cdots \cdots \cdots \\
& -\Delta u^{m}=H_{v^{m}}, \\
& -\Delta v^{m}=H_{u^{m}}, \\
& -\Delta w^{1}=H_{w^{1}}, \\
& \ldots \ldots \ldots \ldots \ldots \\
& -\Delta w^{r}=H_{w^{r}},
\end{aligned}
$$

where $H=H\left(u^{1}, \ldots, u^{m}, v^{1}, \ldots, v^{m}, w^{1}, \ldots, w^{r}\right), H(0, \ldots, 0)=0$, is critical if and only if

$$
a^{\alpha} u^{\alpha} H_{u^{\alpha}}+\left(1-a^{\alpha}\right) v^{\alpha} H_{v^{\alpha}}+\frac{1}{2} w^{\alpha} H_{w^{\alpha}}=\frac{n}{n-2} H,
$$

where $\alpha=1, \ldots, m$ and $\beta=1, \ldots, r$.

\section{Hyperbolic Hamiltonian systems}

Theorem 10. The nonlinear hyperbolic system of Hamiltonian type

$$
\begin{aligned}
& u_{t t}-\Delta u+H_{v}(u, v)=0, \\
& v_{t t}-\Delta v+H_{u}(u, v)=0
\end{aligned}
$$

is critical if and only if

$$
a u H_{u}+(1-a) v H_{v}=\frac{n+1}{n-1} H .
$$

This result is obtained by the same argument as before and we omit the corresponding details pointing out that in the particular case of power nonlinearity

$$
H=\frac{1}{q+1}|u|^{q-1} u+\frac{1}{p+1}|v|^{p-1} v
$$

the latter condition reads

$$
\frac{1}{p+1}+\frac{1}{q+1}=\frac{n-1}{n+1} .
$$

The Pokhozhaev's identity corresponding to the hyperbolic Hamiltonian system in Theorem 10 was obtained in [13] using the Noetherian approach. For a discussion on specific numbers concerning the scalar case see $[1,2]$.

\section{Unbounded Hamiltonian systems}

Theorem 11. The system

$$
\begin{aligned}
& u_{t}-\Delta u=H_{v}(u, v), \\
& -v_{t}-\Delta v=H_{u}(u, v)
\end{aligned}
$$

is critical if and only if

$$
a u H_{u}+(1-a) v H_{v}=\frac{n+2}{n} H .
$$


Again this result as well as the corresponding Pokhozhaev's identity [13] is obtained by the same arguments as before and we omit further details. We observe that in the particular case of power nonlinearity

$$
H=\frac{1}{q+1}|u|^{q-1} u+\frac{1}{p+1}|v|^{p-1} v
$$

the criticality condition reads

$$
\frac{1}{p+1}+\frac{1}{q+1}=\frac{n}{n+2}
$$

The latter condition appears in [16], see also [17].

\section{Acknowledgements}

We wish to thank the referees for their useful suggestions. Yuri Bozhkov is grateful to the Organizers of the 7th International Conference "Symmetry in Nonlinear Mathematical Physics", June 24-30 2007, Kyiv, Ukraine, for having given him the opportunity to present a talk on this subject. He would also like to thank FAPESP, CNPq and FAEPEX-UNICAMP, Brasil, as well as ICTP, Trieste, Italy, for financial support. Enzo Mitidieri acknowledges the support of INTAS-05-100000B-792.

\section{References}

[1] Anco S.C., Ivanova N.M., Conservation laws and symmetries of semilinear radial wave equations, J. Math. Anal. Appl., to appear, math-ph/0608037.

[2] Anco S.C., Liu S., Exact solutions of semilinear radial wave equations in $n$ dimensions, J. Math. Anal. Appl. 297 (2004), 317-342, math-ph/0309049.

[3] Baouendi M.S., Sur une classe d'operateurs elliptique dégénérés, Bull. Soc. Math. France 95 (1967), 45-87.

[4] Bliss G., An integral inequality, J. London Math. Soc. 5 (1930), 40-46.

[5] Bluman G.W., Kumei S., Symmetries and differential equations, Springer, New York, 1989.

[6] Bozhkov Y.D., Noether symmetries and critical exponents, SIGMA 1 (2005), 022, 12 pages, nlin.SI/0511058.

[7] Bozhkov Y.D., Divergence symmetries of semilinear polyharmonic equations involving critical nonlinearities, J. Differential Equations 225 (2006), 666-684.

[8] Bozhkov Y.D., Freire I.L., Group classification of semilinear Kohn-Laplace equations, Nonlinear Anal., to appear.

[9] Bozhkov Y.D., Freire I.L., Divergence symmetries of critical Kohn-Laplace equations on Heisenberg groups, Diff. Uravn., to appear.

[10] Bozhkov Y.D., Gilli Martins A.C., On the symmetry group of a differential equation and the LiouvilleGelfand problem, Rendiconti dell'Istituto di Matematica dell'Università di Trieste 34 (2002), 103-120.

[11] Bozhkov Y.D., Gilli Martins A.C., Lie point symmetries and exact solutions of quasilinear differential equations with critical exponents, Nonlinear Anal. 57 (2004), 773-793.

[12] Bozhkov Y.D., Gilli Martins A.C., Lie point symmetries of the Lane-Emden system, J. Math. Anal. Appl. 294 (2004), 334-344.

[13] Bozhkov Y.D., Mitidieri E., The Noether approach to Pokhozhaev's identities, Mediterr. J. Math., to appear.

[14] Caffarelli L.A., Gidas B., Spruck J., Asymptotic symmetry and local behavior of semilinear elliptic equations with critical Sobolev growth, Comm. Pure Appl. Math. 42 (1989), 271-297.

[15] Clément P., de Figueiredo D. G., Mitidieri E., Quasilinear elliptic equations with critical exponents, Topol. Methods Nonlinear Anal. 7 (1996), 133-170.

[16] Clément P., Felmer P., Mitidieri E., Homoclinic orbits for a class of infinite-dimensional Hamiltonian systems, Ann. Scuola Norm. Sup. Pisa Cl. Sci. (4) 24 (1997), 367-393. 
[17] Clément P., van der Vorst R.C.A.M., On the non-existence of homoclinic orbits for a class of infinitedimensional Hamiltonian systems, Proc. Amer. Math. Soc. 125 (1997), 1167-1176.

[18] D'Ambrosio L., Hardy inequalities related to Grushin type operators, Proc. Amer. Math. Soc. 132 (2004), 725-734.

[19] Garofalo N., Lanconelli E., Existence and nonexistence results for semilinear equations on the Heisenberg group, Indiana Univ. Math. J. 41 (1992), 71-98.

[20] Gidas B., Spruck J., Global and local behavior of positive solutions of nonlinear elliptic equations, Comm. Pure Appl. Math. 34 (1981), 525-598.

[21] Grushin V.V., On a class of hypoelliptic operators, Math. Sbornik USSR 12 (1970), 458-476.

[22] Guedda M., Veron, L., Quasilinear elliptic equations involving critical Sobolev exponents, Nonlinear Anal. 13 (1989), 879-902.

[23] Ibragimov N.H., Noether's identity, Dinamika Sploshnoy Sredy 38 (1979), 26-32 (in Russian).

[24] Ibragimov N.H., Transformation groups applied to mathematical physics, D. Reidel Publishing Co., Dordrecht, 1985.

[25] Mitidieri E., A Rellich identity and applications, Rapporti Interni, Univ. Udine (1990), no. 25, 1-35.

[26] Mitidieri E., A Rellich type identity and applications, Commun. Partial Differential Equations 18 (1993), 125-151.

[27] Mitidieri E., Nonexistence of positive solutions of semilinear elliptic systems in $R^{N}$, Differential Integral Equations 9 (1996), 465-479.

[28] Mitidieri E., Pokhozhaev S.I., A priori estimates and the absence of solutions of nonlinear partial differential equations and inequalities, Proc. Steklov Inst. Math. 234 (2001), 1-362.

[29] Olver P.J., Applications of Lie groups to differential equations, Springer, New York, 1986.

[30] Ovsiannikov L., Group analysis of differential equations, Academic Press, London, 1982.

[31] Pokhozhaev S.I., On the eigenfunctions of the equation $\Delta u+\lambda f(u)=0$, Dokl. Akad. Nauk SSSR 165 (1965), 36-39 (English transl.: Soviet Math. Dokl. 6 (1965), 1408-1411).

[32] Pokhozhaev S.I., On eigenfunctions of quasilinear elliptic problems, Mat. Sb. 82 (1970), 192-212 (English transl.: Math. USSR Sbornik 11 (1970), 171-188).

[33] Pohozaev S.I., Veron L., Nonexistence results of solutions of semilinear differential inequalities on the Heisenberg group, Manuscripta Math. 102 (2000), 85-99.

[34] Pucci P., Serrin S., A general variational identity, Indiana Univ. Math. J. 35 (1986), 681-703.

[35] Pucci P., Serrin S., Critical exponents and critical dimensions for polyharmonic operators, J. Math. Pures Appl. (9) 69 (1990), 55-83.

[36] Serrin J., Zou H., Non-existence of positive solutions of semilinear elliptic systems, Discourses Math. Appl. 3 (1994), 55-68.

[37] Serrin J., Zou H., Non-existence of positive solutions of the Lane-Emden systems Differential Integral Equations 9 (1996), 635-653.

[38] Serrin J., Zou H., Existence of positive solutions of the Lane-Emden systems, Atti Sem. Mat. Fis. Univ. Modena 46 (1998), supl., 369-380.

[39] van der Vorst R.C.A.M., Variational identities and applications to differential systems, Arch. Rational Mech. Anal. 116 (1992), 375-398. 